\title{
UMA PROPOSTA DE OBJETO DE APRENDIZAGEM PARA ENSINO DA INTERAÇÃO DOS CAMPOS MAGNÉTICOS ENTRE POLOS DE NOMES DIFERENTES USANDO REALIDADE AUMENTADA
}

\author{
Suzana da Hora Macedo, Instituto Federal Fluminense, PGIE/UFRGS \\ shmacedo@iff.edu.br \\ Filipe Arantes Fernandes, Centro Universitário São José de Itaperuna \\ filipearantes@fsj.edu.br \\ José Valdeni de Lima, PGIE/UFRGS \\ valdeni@inf.ufrgs.br \\ Maria Cristina Villanova Biazus, PGIE/UFRGS \\ cbiazus@ufrgs.br
}

\begin{abstract}
Resumo
Propõe-se neste trabalho apresentar um Objeto de Aprendizagem para o ensino da interação dos campos magnéticos entre dois pólos de nomes diferentes desenvolvido em ambiente de Realidade Aumentada. No ambiente criado em Realidade Aumentada há a presença simultânea de objetos reais e virtuais. Neste ambiente são visualizados os campos magnéticos de dois ímãs, demonstrando-se a sua interação. Neste Objeto de Aprendizagem o aluno pode visualizar esta interação em 3D e interagir com os campos. Este Objeto de Aprendizagem pode servir de apoio à aula convencional no estudo dos campos magnéticos dos ímãs. Foram feitas análises e avaliações para a conclusão do trabalho, destacando-se as suas vantagens no processo de aprendizagem.
\end{abstract}

Palavras-chave: Objetos de aprendizagem, simulação, campos magnéticos, realidade aumentada.

\section{A PROPOSAL OF LEARNING OBJECT TO TEACH THE INTERACTION BETWEEN MAGNETIC FIELDS OF POLES OF DIFFERENT NAMES USING AUGMENTED REALITY}

It is proposed in this paper to present a Learning Object for teaching the interaction of magnetic fields between the two poles of different names developed in Augmented Reality environment. In the environment created in Augmented Reality there is the simultaneous presence of real and virtual objects. In this environment are shown the magnetic fields of two magnets, demonstrating their interaction. In this Learning Object, the student can see this interaction in 3D and interact with the fields. This Learning Object can be used to support the conventional classroom to study the magnetic fields of magnets. Reviews and evaluations were done to complete the work, highlighting its advantages in the learning process.

Keywords: learning objects, simulation, magnetic fields, augmented reality.

\section{Introdução}

Nos cursos técnicos de Eletrotécnica o ensino de Eletromagnetismo é muito importante, pois a compreensão dos fenômenos eletromagnéticos é base científica e 
tecnológica para o estudo das Máquinas Elétricas Girantes que são os fundamentos da conversão de energia mecânica em elétrica e vice-versa. Portanto, estes são conceitos de fundamental importância na formação do técnico em Eletrotécnica.

Verifica-se que as dificuldades de aprendizagem dos conteúdos de Eletromagnetismo se concentram na impossibilidade de visualização dos campos magnéticos por parte dos alunos no espaço tridimensional. Segundo Paz, "Constatamos que as dificuldades de aprendizagem dos conteúdos de Eletromagnetismo se concentram no entendimento das interações e comportamento das variáveis eletromagnéticas no espaço tridimensional, [...]" (2007, p. viii).

Neste trabalho, foi elaborado um Objeto de Aprendizagem baseado em ambiente de Realidade Aumentada que proporcionará ao estudante interatividade e visualização em 3D da interação entre campos magnéticos de pólos de nomes diferentes. Segundo Guillermo et al. (2005, p.2), “... as simulações tem se destacado como ótimos objetos de aprendizagem, principalmente na área das engenharias, ou até das ciências exatas." Este objeto servirá para ampliar o horizonte do aluno, possibilitando a interação com o fenômeno físico em estudo, fazendo com que ele visualize no mundo virtual objetos que os seres humanos não conseguem visualizar no mundo real.

Segundo Kaufmann et al. (2005), a principal vantagem do uso da Realidade Aumentada é que os alunos realmente vêem objetos tridimensionais os quais até agora tinham que calcular e construir com os métodos tradicionais - principalmente papel e caneta.

No estudo da interação entre o campo magnético de pólos de nomes diferentes é geralmente apresentada ao estudante a figura 1 ou similar, onde se pode visualizar de forma bidimensional a interação entre esses campos magnéticos.

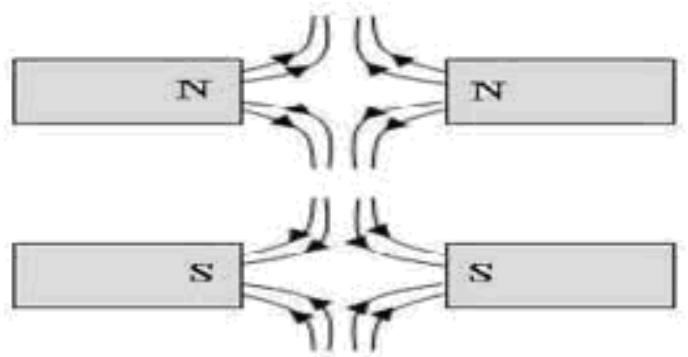

Figura 1. Interação entre campos magnéticos de pólos de nomes diferentes

Fonte: Educação Uol. Silva, João Freitas da

http://educacao.uol.com.br/fisica/campo-magnetico---representacao-geometrica.jhtm

Nota-se que esta visualização é muito rudimentar, principalmente porque apresenta uma visualização bidimensional e os campos magnéticos são tridimensionais. Neste sentido, a informática pode ser uma grande aliada do professor. "O conhecimento adquire novas representações, ... seja através de simulações, que permitem vivenciar de modo virtual situações, cuja forma real muitas vezes não se percebe ..." (Costa, 2004, p.17)

Com o apoio da Realidade Aumentada o aluno pode ver em terceira dimensão a interação entre os campos magnéticos de pólos de nomes diferentes de dois ímãs. Tal fenômeno não é possível de ser visto a olho nu, pois os campos magnéticos não são visíveis pelo olho humano. De acordo com Barros (2007, p.119), “... professor, aluno, classe, estão todos envolvidos no tema de modo participativo, imersos no ambiente virtual construído para cada objetivo." Neste sentido, a Realidade Aumentada servirá 
para ampliar o horizonte do estudante, onde ele poderá visualizar em terceira dimensão e também interagir com os campos magnéticos estudados.

\section{Materiais e métodos}

\subsection{Como funciona a Realidade Aumentada}

A Realidade Aumentada, a partir de filmar uma cena em tempo real, e a partir de um marcador presente na cena, traz para a tela do computador uma cena em um mundo virtual misturado com um mundo real, que é o mundo em Realidade Aumentada. A formação do ambiente em Realidade Aumentada é exemplificada na figura 2.

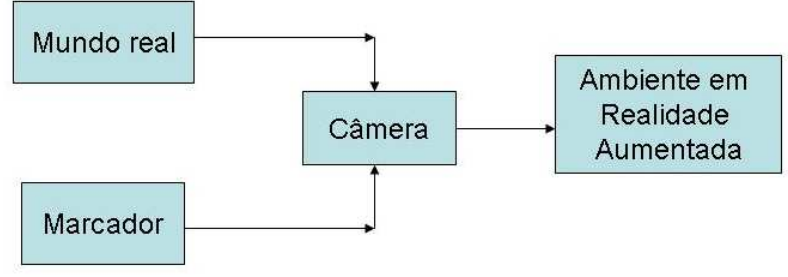

\section{Figura 2. Formação do mundo em Realidade Aumentada.} Fonte: Macedo et al (2010).

De acordo com Coelho e Bähr (2005), o ambiente em Realidade Aumentada é formado a partir da mistura da filmagem de um certo local com cenas de um mundo virtual. Estas cenas formadas devem dar a impressão de que objetos virtuais existam no mundo real.

Segundo Macedo et al (2010), a Realidade Aumentada funciona da seguinte maneira:

1-Coloca-se um marcador em um objeto onde se deseja que ocorra a superposição do objeto virtual;

2-Esse marcador será visualizado pela câmera do microcomputador;

3-Se o mesmo for reconhecido, levará a uma biblioteca pré-estabelecida;

4-Surgirá na tela do computador o primeiro objeto onde estava localizado o marcador, juntamente com o objeto que estará na biblioteca.

5-Os dois objetos serão fundidos em um mundo misto que misturará o mundo real com o mundo virtual.

\subsection{Como foi realizado este experimento}

Este Objeto de Aprendizagem foi elaborado utilizando-se a ferramenta SACRA (Sistema de Autoria Colaborativa com Realidade Aumentada). O sistema SACRA foi desenvolvido em 2008 pelo aluno de mestrado Rafael Santin sob orientação do professor Cláudio Kirner. Segundo Kirner (2010), a partir da dificuldade que os leigos em computação tinham em trabalhar com ARTool Kit, esta ferramenta foi desenvolvida 
para permitir que usuários não especialistas em computação pudessem desenvolver aplicações com uma ferramenta mais simples.

$\mathrm{Na}$ seqüência será explicado o experimento que foi realizado pelo desenvolvedor. Inicialmente é apresentado o primeiro marcador que se encontra na figura 3 denominado "referência" para que se dê início ao processo.

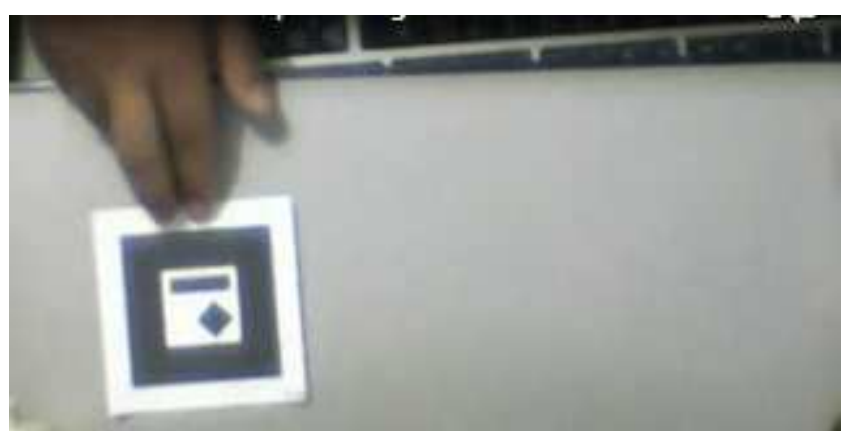

Figura 3. Marcador referência sendo apresentado.

Surge a esfera apresentada na figura 4, correspondendo ao marcador apresentado.

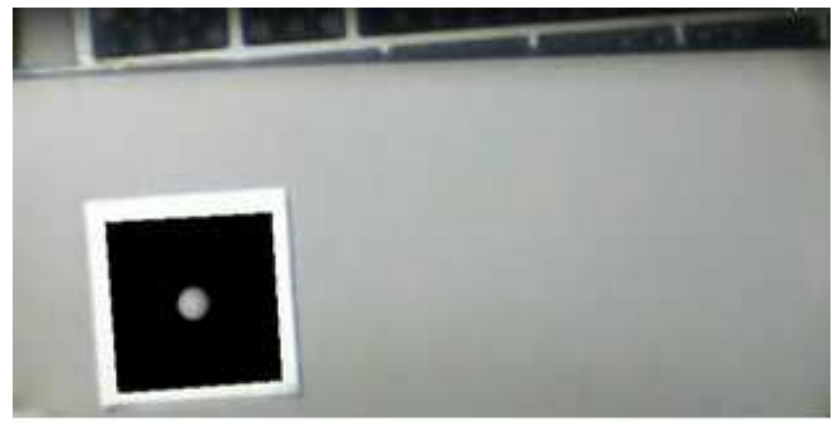

Figura 4. Esfera.

É aproximado então um segundo marcador, chamado de marcador "inspeção", que se encontra na figura 5. Surgirá então uma segunda esfera. Esta deverá ser encaixada na primeira para que se dê seqüência à programação.

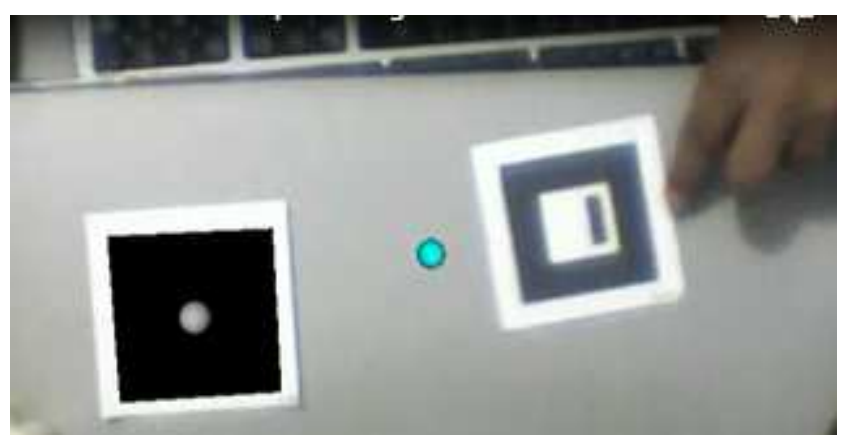

Figura 5. Aproximação do Segundo marcador com a segunda esfera.

Quando ocorre o encaixe entre as duas esferas, surge então o primeiro ímã, como pode ser visto na figura 6 . 


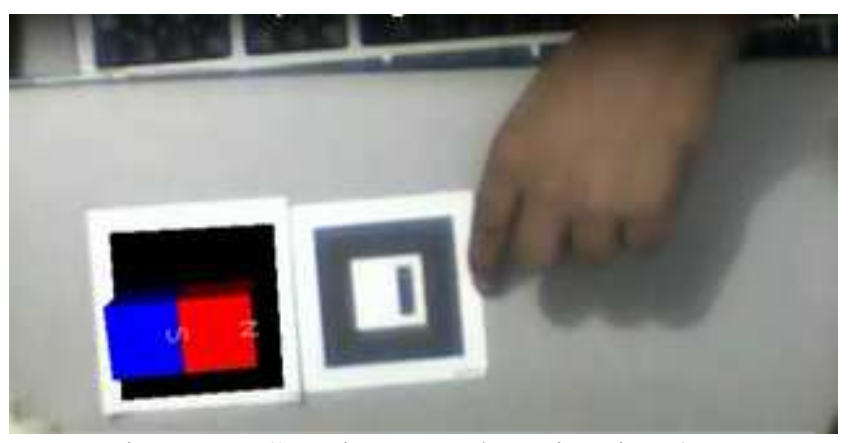

Figura 6. Surgimento do primeiro ímã.

Este ímã em ambiente de Realidade Aumentada pode ser manipulado livremente pelo usuário em terceira dimensão. Nas figuras 7 e 8, pode-se vê-lo em outra posição.

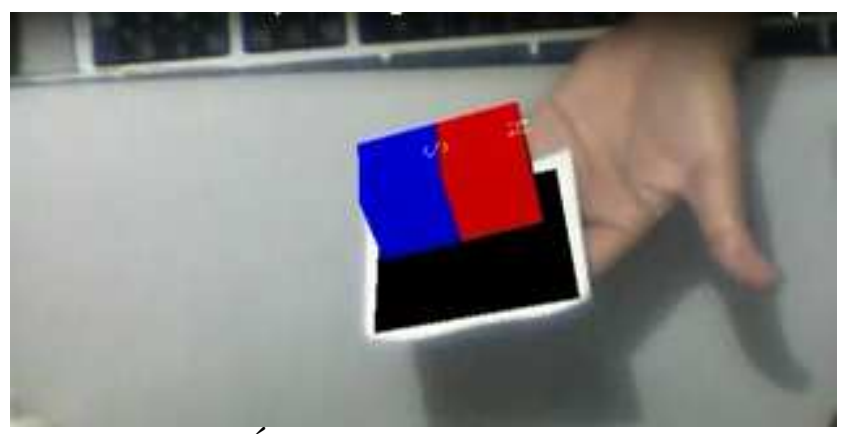

Figura 7. Ímã em uma segunda posição.

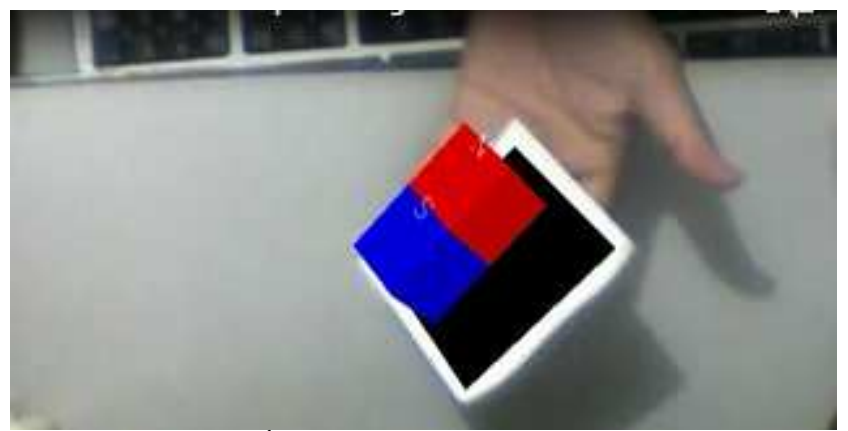

Figura 8. Ímã em uma terceira posição.

É aproximado o terceiro marcador, denominado "controle", demonstrado na figura 9, que definirá as próximas ações que serão desencadeadas na programação.

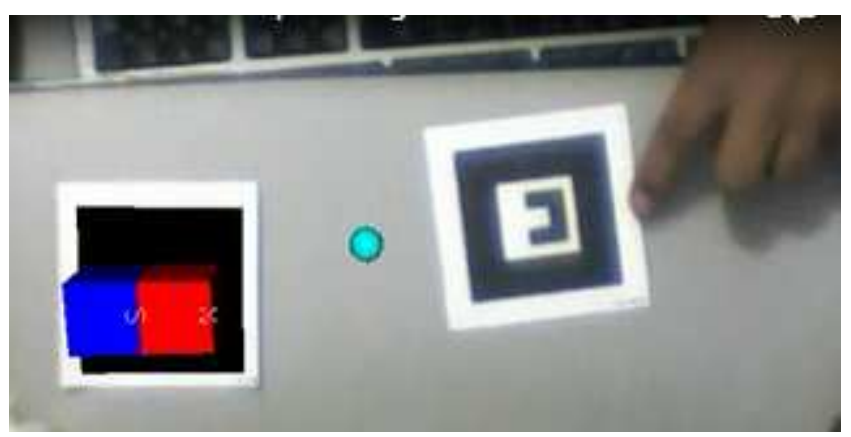

Figura 9. Marcador "controle" sendo aproximado. 
Surge o campo magnético do primeiro ímã, demonstrado na figura 10 , como estava programado pela ação definida pelo marcador controle.

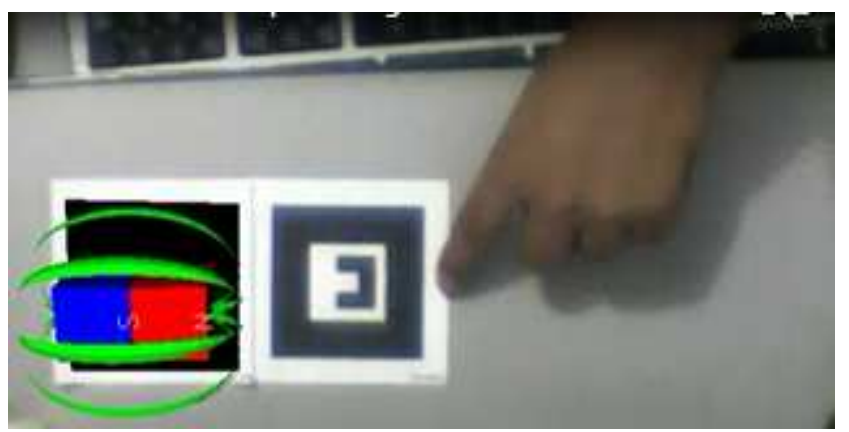

Figura 10. Campo magnético do primeiro ímã.

Este campo magnético também pode-ser manipulado livremente pelo usuário como pode ser verificado nas figuras $11,12,13$ e 14 .

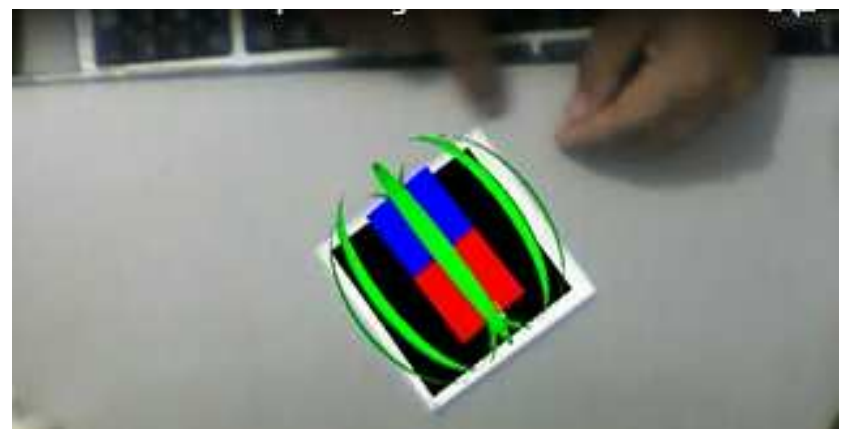

Figura 11. Campo magnético em uma segunda posição.

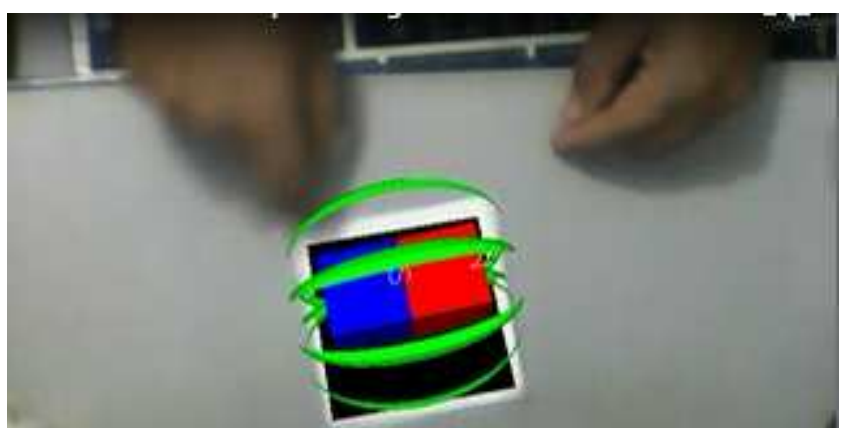

Figura 12. Campo magnético em uma terceira posição.

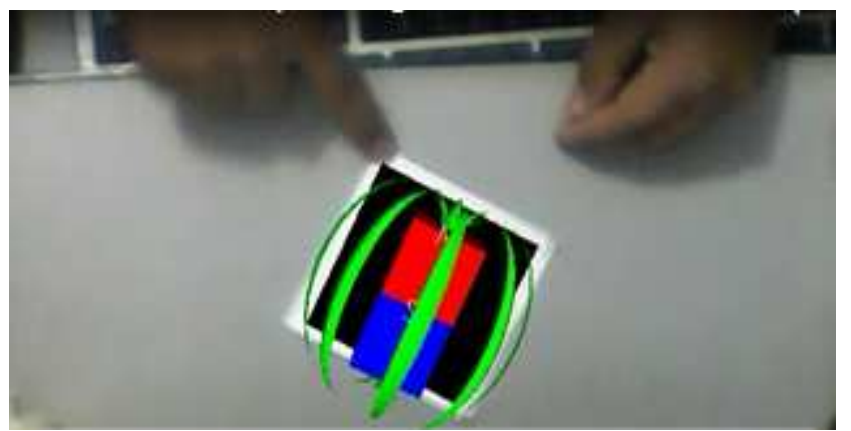

Figura 13. Campo magnético em uma quarta posição. 


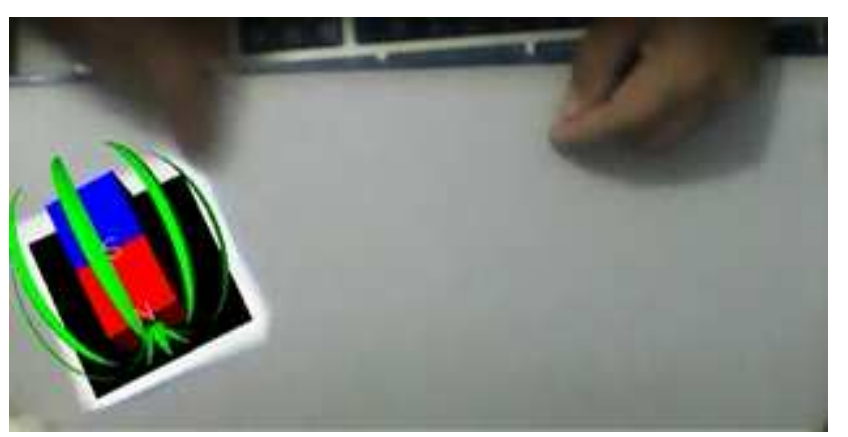

Figura 14. Campo magnético em uma quinta posição.

A seguir é aproximado o marcador "controle", na figura 15, novamente para que se dê seqüência à programação.

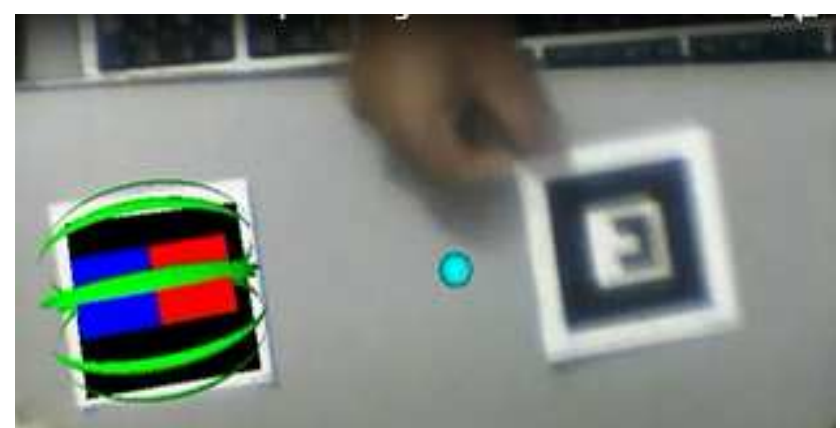

Figura 15. Marcador "controle” sendo aproximado.

Quando o marcador "controle" é apresentado à câmera, o mesmo traz a imagem do campo magnético do segundo ímã já representando a repulsão que ocorrerá entre os mesmos, como pode ser visto na figura 16.

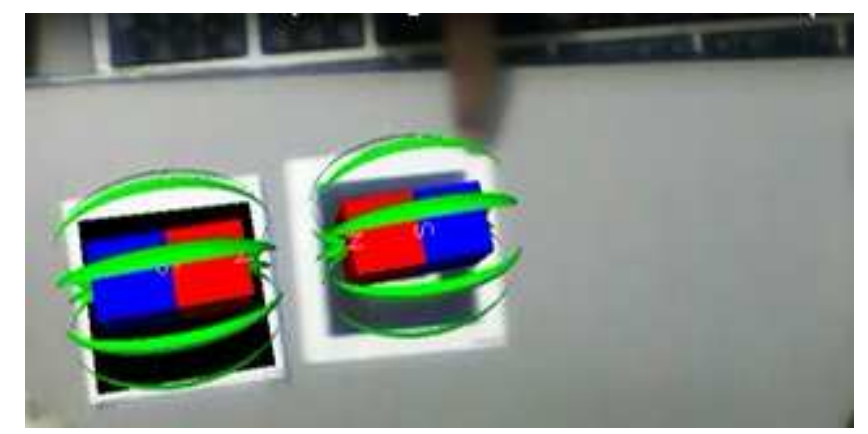

Figura 16. Interação entre os campos magnéticos: repulsão

$\mathrm{Na}$ figura 17 pode-se perceber estes campos magnéticos em uma segunda posição, podendo haver a manipulação por parte do usuário. 


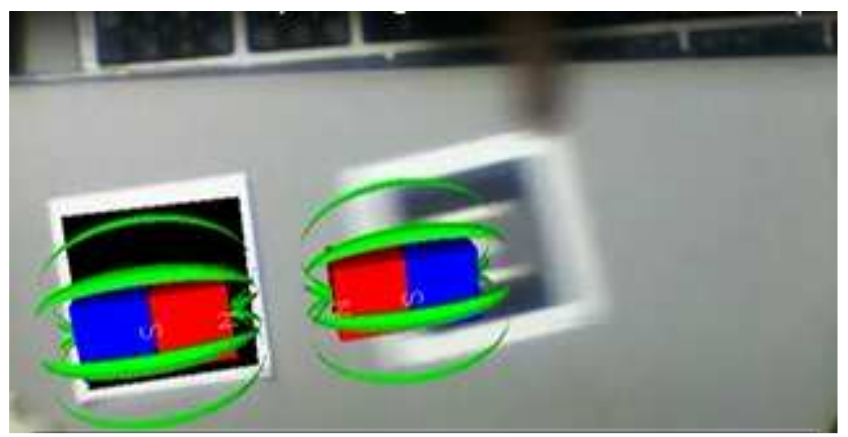

Figura 17. Interação entre os campos magnéticos em uma segunda posição

\subsection{Uma experiência em sala de aula}

O objetivo deste experimento foi criar um ambiente lúdico em sala de aula, onde o aluno pudesse interagir com o Objeto de Aprendizagem e também pudesse visualizar o mesmo, aumentando também a motivação do aluno com o objetivo de aprender. $\mathrm{O}$ aluno pode visualizar a interação entre dois campos magnéticos de pólos de nomes diferentes como nunca experimentado anteriormente. Neste experimento o aprendiz teve a oportunidade de visualizar esta interação entre os campos magnéticos em terceira dimensão.

Foi feito um teste preliminar no mês de maio de 2011, com cinco alunos do curso de Eletrotécnica, turno noturno, utilizando este experimento em sala de aula. Foi utilizado um laptop e também um data show. Inicialmente o experimento foi realizado pelo professor e os alunos puderam assistir no data show. A seguir os cinco alunos tiveram a oportunidade de testá-lo individualmente. Este pequeno teste foi realizado com o objetivo de enriquecimento do Objeto de Aprendizagem para posterior uso como apoio às aulas convencionais. Os resultados serão demonstrados e discutidos a seguir.

\section{Resultados e discussão}

No teste realizado, os cinco alunos responderam, quando questionados, que conseguiram sim, visualizar a interação entre os campos magnéticos de pólos diferentes.

Quando perguntados se acharam fácil usar Realidade Aumentada, 3 alunos responderam que sim e dois disseram ter dificuldade com uso dos marcadores.

À pergunta: "Você gostaria de ter mais aulas usando Realidade Aumentada?", todos os 5 alunos responderam que gostariam.

$\mathrm{Na}$ questão "Escreva neste espaço as suas observações e sugestões", onde os alunos puderam escrever livremente, foram verificadas as seguintes respostas:

(a) (20 anos - homem) "Gostaria de ter mais aulas com essa Realidade Aumentada."

(b) (25 anos - homem) "Seria útil usar mais vezes, quando necessário."

(c) (25 anos - homem) "Gostei porque você vê que a teoria existe de verdade."

(d) (22 anos - homem) "Tem que ser mais utilizada, porque os benefícios serão enormes."

(e) (19 anos - homem) "As aulas ficam mais interessantes. É mais legal, pois vemos como funciona na realidade."

O Objeto de Aprendizagem em Realidade Aumentada proposto visou apresentar aos alunos uma forma de interagir e visualizar em três dimensões a interação entre os 
campos magnéticos de pólos de nomes diferentes. Com este trabalho, deseja-se propor uma nova forma de ensinar campos magnéticos. Com este experimento, e de acordo com o questionário respondido pelos alunos, pode se verificar que os alunos conseguiram visualizar e interagir com os campos magnéticos de pólos diferentes, além de terem gostado de utilizar o Objeto de Aprendizagem baseado em Realidade Aumentada. Também, todos gostariam de ter mais aulas utilizando Realidade Aumentada. Segundo as perguntas em que puderam responder livremente, comentaram que acharam útil, as aulas ficam mais interessantes e que ajuda a compreender a teoria.

\section{Conclusões}

Pelo que foi demonstrado, os alunos conseguiram realmente visualizar em 3D os campos magnéticos de pólos diferentes e também puderam interagir com estes campos.

Alguns alunos tiveram dificuldade com o uso dos marcadores. Deve-se pensar em melhorar a explicação sobre o uso dos mesmos, inclusive distribuindo folhetos explicativos.

A Realidade Aumentada apresenta as seguintes vantagens, entre outras:

- Permitiu aos alunos a visualização da interação dos campos magnéticos de pólos diferentes;

- Permitiu a interação do aluno com estes campos magnéticos;

- A partir do momento em que o Objeto de Aprendizagem em Realidade Aumentada foi elaborado, sua utilização é simples e prática;

- Simplicidade e economia do equipamento utilizado: o experimento foi realizado com apenas um laptop com uma webcam.

- O ambiente em Realidade Aumentada é excelente para visualização da interação entre os campos magnéticos.

Conclui-se, portanto, que, com este Objeto de Aprendizagem, as contribuições para as aulas convencionais serão de grande valia.

\section{Referências bibliográficas}

BARROS, Nelci Moreira. Aprendizagem a Distancia. Do Rádio Ilustrado à Realidade Virtual Aumentada. Florianópolis, SC: Insular, 2007.

COELHO, A. H.; Bähr, H. P. Visualização de Dados CAD e LIDAR por Meio de Realidade Aumentada. In: XII Simpósio de Sensoriamento Remoto, 16-21 de abril de 2005, INPE, pp. 2925-2932. Disponível em:

<http://marte.dpi.inpe.br/col/ltid.inpe.br/sbsr/2004/11.11.08.15/doc/2925.pdf>. Acesso em 03 abr. 2011.

COSTA, José Wilson da; PAIM, Isis. Informação e conhecimento no processo educativo. In: Costa, José Wilson; Oliveira, Maria Auxiliadora Monteiro (Org.). Novas Linguagens e Novas Tecnologias. Petrópolis, RJ: Vozes, 2004. 
GUILLERMO, Oscar Eduardo Patrón; TAROUCO, Liane Margarida Rockenbach; ENDRES, Luiz Augusto Magalhães. Desenvolvimento de Objetos Educacionais: Experimentos em Hidráulica. RENOTE - Revista Novas Tecnologias na Educação, Porto Alegre, v. 3 N², novembro, 2005.

KAUFMANN, H; STEINBÜEGL, K.; DÜNSER, A; GLÜCK, J. General Training of Spatial Abilities by Geometry Education in Augmented Reality. In: Annual Review of CyberTherapy and Telemedicine: A Decade of VR, vol. 3, pp. 65-76, 2005. Disponível em:

<http://www.ims.tuwien.ac.at/media/documents/publications/CT05_GeomEdu_Spatial Abilities.pdf> Acesso em 27 jul. 2010.

KIRNER, C. Funcionamento e utilização do Sistema de Autoria Colaborativa com Realidade Aumentada. 2010. <Disponível em: http://www.ckirner.com/sacra/>. Acesso em 04 abr. 2011.

MACEDO, S. H.; LIMA, J. V.; AZEVEDO, F. C. Uso da Realidade Aumentada no Ensino de Sólidos. In: Congresso Iberoamericano de Informática na Educação. Santiago: Universidad de Chile, p. 179-183, 2010.

PAZ, A. M. Atividades Experimentais e Informatizadas: Contribuições para o Ensino de Eletromagnetismo. Universidade Federal de Santa Catarina, Florianópolis, 2007. 228 p. Tese de Doutorado. 